\title{
Ultrasonography-guided PNL in comparison with laparoscopic ureterolithotomy in the management of large proximal ureteral stone
}

Hossein Karami, Mohammad Mohsen Mazloomfard, Behzad Lotfi, Asghar Alizadeh, Babak Javanmard

Urology and Nephrology Research Center (UNRC), Shohada Medical Center, Shahid Beheshti University, M.C. (SBMU), Tehran, I.R. Iran

\section{ABSTRACT}

Purpose: The aim of study was to evaluate the clinical outcomes of PNL in comparison with laparoscopic ureterolithotomy (LUL) in proximal ureteral stones larger than $1 \mathrm{~cm}$. Materials and Methods: A total of 80 patients who were candidates for treatment of large ureteral stones in our urology center were enrolled in the study between September 2004 and September 2008. By using patient randomization, they were assigned into two forty-patient groups (PNL and LUL). After evaluating the patients with laboratory tests and IVP, PNL was performed under sonography guidance in the prone position or the patients were submitted to classic laparoscopic ureterolithotomy (LUL) transperitoneally. All patients underwent postoperative assessments including KUB and ultrasonography.

Results: A hundred-percent success was achieved in both groups. The mean age of the patients were 39.4 (16-63) and 35.2 (18-57) years old in PNL and LUL groups, respectively. The mean stone size in PNL group was $14.2(10-25) \mathrm{mm}$ and in LUL group was 13.5 (10-28) $\mathrm{mm}$. The duration of the operations were 54.35 (50-82) minutes, and 82.15 (73-180) minutes $(\mathrm{P}<0.0001)$; and the average hospital stay days were $2.6(2-5)$ and $3.5(3-8)$ days ( $p=0.011$ ) in groups PNL and LUL, accordingly. The mean Hb decrease in PNL group was $0.9 \mathrm{mg} / \mathrm{dL}$ and in LUL group was $0.4 \mathrm{mg} / \mathrm{dL}(\mathrm{p}=0.001)$. No statistically significant differences in terms of blood transfusion, fever, ICU admission, and prolonged urinary leakage were detected in both groups.

Conclusion: According to our study, percutaneous nephrolithotomy under ultrasonography guidance is comparable with the laparoscopic ureterolithotomy for the treatment of proximal ureteral stones larger than $1 \mathrm{~cm}$.

\section{ARTICLE INFO}

\section{Key words:}

Urinary Calculi; Nephrostomy, Percutaneous; Laparoscopy; Ureteral Calculi

Int Braz J Urol. 2013; 39: 22-9

Submitted for publication: March 12, 2012

Accepted after revision: August 19, 2012

\section{INTRODUCTION}

Ureteral stones can cause obstructive uropathy and subsequent deterioration of renal function (1). Since the patient's symptoms and stone size are not good predictors of renal function loss, and there is no clear time threshold for irreversible damage, intervention should be strongly conside- red in any patient with ureteral obstruction unless close monitoring of renal function is available (2).

An impacted stone is defined as a stone that cannot be bypassed either by a wire, or a catheter, or a stone remaining at the same site in the ureter for over two months (3). The treatment options for these stones consist of extracorporeal shockwave lithotripsy (ESWL), ureteroscopic pro- 
cedures, percutaneous nephrolithotomy (PNL), laparoscopic ureterolithotomy (LUL), and open stone surgery (OSS) (2).

However, OSS has been associated with longer hospitalization and greater postoperative morbidity (4), and the efficacy of ESWL drops significantly for impacted stones larger than $1 \mathrm{~cm}$ in diameter (5). Several reports have suggested that ureteroscopy should be the primary approach for the impacted ureteral stones, especially with flexible ureteroscopic lithotripsy using the holmium laser (6-8).

We frequently encounter proximal ureteral stones larger than $1 \mathrm{~cm}$ at our center and a significant proportion of them are impacted or failed to be removed by previous ESWL attempts. Altogether, holmium laser lithotripsy has good results with low complications (7), but it is expensive and unavailable in many centers. The use of pneumatic lithotripsy instead of holmium laser is not appropriate because of high possibility of stone migration (9).

Alternatively, PNL is an option for large proximal ureteral stones, with a reported median stone-free rate of $86 \%$. The proximal dilated ureter makes most of these stones approachable with a rigid nephroscope or large-caliber flexible instruments that can fragment and remove large stone burdens efficiently (9). Traditionally, PNL has been performed in the prone position and mostly relies on fluoroscopy, exposing both the patient and the surgical team to radiation. Recently, some investigations showed that PNL under ultrasonography guidance is a safe and convenient procedure. Therefore, this study was designed to compare ultrasonography guided PNL in the prone position with LUL as two minimally invasive techniques in the management of proximal ureteral stones larger than $1 \mathrm{~cm}$.

\section{MATERIALS AND METHODS}

A total of 80 patients with proximal ureteral stones (above the inferior margin of kidney) and larger than $1 \mathrm{~cm}$ at our urology center were enrolled in the study between September 2004 and September 2008. Patients with kidney anomalies, severe skeletal deformities, uncontrolled coagulo- pathies, and the previous histories of PNL or open renal stone surgeries were excluded. These patients were assigned to one of the two treatments in a randomized sequential order, with 40 patients in each group (PNL or laparoscopic ureterolithotomy (LUL)). Some information such as demographic data including age, sex, stone laterality and stone size (the mean diameter) in these patients were recorded. Preoperative evaluation consisted of tests such as urine analysis, urine culture and renal function tests. In patients with active urinary infection, appropriate treatments were performed. Excretory urography (IVU) was the primary imaging modality in all patients. The stone burden was determined by multiplying the stone length by the stone width in centimeters, as measured on the preoperative plain abdominal film, or on IVU in the cases of radiolucent stones.

The patients were fully advised on the nature of the study, and the informed consents were obtained. The design of this research was approved by the bioethics board of the Urology and Nephrology Research Center (UNRC).

\section{Operation technique}

Both procedures were performed under general anesthesia.

In the PNL group, after introducing an open ended $6 \mathrm{~F}$ ureteral catheter in a lithotomic position, the patient was repositioned into a standard prone position with all pressure points padded. The ureteral catheter was used for continuous and rapid injection of normal saline during lithotripsy to clear out calculi fragments located at the ureter. Detection of the pyelocaliceal system was achieved sonographically with a 3.5-MHz probe through the posterior abdominal wall. An 18-gauge access needle was inserted through the fornix, usually at the posterior axillary line below the 12th rib, targeting the middle calices. With a steady suction on the syringe, access to the collecting system was confirmed when urine was withdrawn. Then, a 0.035 inch $\mathrm{J}$ tipped guide wire was introduced into the targeted calyx. We injected $50 \mathrm{cc}$ normal saline to induce hydronephrosis when it did not exist. The nephrostomy tract was dilated by a one-shot dilatators and an Amplatz sheath (28 to 30F) was placed. All these steps were under 
real-time ultrasonography through the posterior abdominal wall.

By using rigid nephroscope and Swiss pneumatic lithotripsy, stones were fragmented and extracted by grasping forceps. Clean pressurized air at 0.35 to $0.5 \mathrm{Mpa}$ was used as an energy source to fire the projectile onto a metal rod that was in contact with the stone. If the location of the access was improper, then access was achieved under fluoroscopic guidance and the ultrasonography-guided access was termed a failure. After stone removal and final nephroscopy control for detecting any residual stone, Amplatz sheets were removed and skin was sutured.

In the laparoscopy group, patients were prescribed oral laxatives and a single dose of a broad-spectrum parenteral antibiotic the day, and one hour before the procedure, respectively. After general anesthesia, a Foley catheter and a nasogastric tube were inserted in all patients. The umbilicus was placed over the break on the operating table and the patient was positioned in a modified lateral decubitus position. The table could be flexed as needed. With the axillary roll and supportive pads at the buttocks and flank, the patient was remained securely in place while the table was rolled toward the surgeon to assist with retraction of the bowel. First $10 \mathrm{~mm}$ trocar for camera was inserted in the upper umbilicus margin under direct vision in the peritoneum. After gas insufflation, the patient was positioned to full flank, and the second $10 \mathrm{~mm}$ port was placed at the level of the umbilicus, lateral to the ipsilateral rectus muscle; then, a $5 \mathrm{~mm}$ port was inserted in the midline between the umbilicus and the xiphoid process. After the insertion of the instruments and the camera, the white line of Toldt was incised and colon mobilized medially, in order to expose the gonadal vessels and the ureter that is usually located just deep to them. Once identified, the ureter was elevated and followed proximally to the stone. Once ureteral stone identified, we hooked the ureter above the stone to prevent its migration. Then, the ureter was incised laterally about $1 \mathrm{~cm}$ with the $5 \mathrm{~mm} \mathrm{~J}$ type electrocautery hook. After manipulation and exertion of stone, it was extracted from one of the trocars site by using a bag. A double pigtail ureteral catheter was placed from one of the trocar sites and the ureterotomy site was closed with absorbable sutures. A drain was inserted from the trocar site intraperitoneally at the end of operation.

In the PNL group, Foley and ureteral catheters were removed on the first post-operation day (POD) unless significant hematuria or ureteral irritation was diagnosed intraoperatively to postpone the removal. The patient was discharged the day after the catheter removal.

In the LUL group, Foley catheter was removed at the 2nd POD and the drain was removed in the 3rd POD and the patient was discharged from hospital on the same day. If drain discharge was over $75 \mathrm{~mL} /$ day, removal of Foley catheter or drain was postponed.

Demographic characteristics, time of operation (from first skin incision to the last skin suture), hospitalization time, change in hemoglobin level (preoperative and the day one after surgery), need for parental pethidine, complications such as perioperative bleeding (need for blood transfusion), postoperative fever (oral temperature $>38^{\circ}$ $\mathrm{C}$ ), prolonged leakage ( $>75 \mathrm{~mL} /$ day), and ICU admission were compared between the two groups.

Ultrasonography and KUB were performed 48 hours after operation to evaluate stone-free status (no residual stone fragments) and residual stone burden. Ureteral stricture was determined by IVU and renal ultrasound studies that were carried out routinely six months after the treatment.

Data analysis was performed by using SPSS software (Statistical Package for the Social Sciences, V. 15; SPSS Inc, Chicago, IL, USA) using Student's t-test and chi-square test. A p-value of less than 0.05 was considered statistically significant.

\section{RESULTS}

Demographic and clinical characteristics of patients are shown in Table-1. The average stone diameter (Min-Max) in PNL group and in LUL group was $14.2 \mathrm{~mm}$ (10-25) and $13.5 \mathrm{~mm}$ (10-28), respectively $(\mathrm{P}$-value $=0.56)$.

In PNL group, ultrasonography guided access was achieved successfully in all cases, but in 6 patients, the exposure was not optimal for approaching the ureteropelvic junction (UPJ). In 
Table 1 - Demographic and clinical characteristics of patients.

\begin{tabular}{lccc}
\hline & LUL $(\mathrm{n}=40)$ & PNL $(\mathrm{n}=40)$ & P value \\
\hline Mean age (years) & $35.2(18-57)$ & $39.4(16-63)$ & 0.21 \\
Male (\%) & $24(60)$ & $28(70)$ & 0.48 \\
Previous history of ESWL (\%) & $4(10)$ & $8(20)$ & 0.35 \\
Impacted Stone (\%) & $17(42.5)$ & $10(25)$ & 0.16 \\
Stone location & & & 0.65 \\
Right side (\%) & $21(52.5)$ & $24(60)$ & \\
Left side (\%) & $19(47.5)$ & $16(40)$ & \\
Hydronephrosis & & $5(12.5)$ & \\
Mild (\%) & $6(15)$ & $12(30)$ & 0.67 \\
Moderate (\%) & $15(37.5)$ & $23(57.5)$ & 0.56 \\
Severe (\%) & $19(47.5)$ & $14.2(10-25)$ & \\
Stone diameter & $13.5(10-28)$ & & \\
\hline
\end{tabular}

that situation, after injecting the contrast medium into the collecting system, another access was created under fluoroscopy control. When moderate to severe hydronephrosis was present, optimal exposure to UPJ was possible even from the lower pole by gently turning the rigid nephroscope and Amplatz sheath. In 25 patients, the stone was fragmented first, and extracted subsequently; for the remaining cases stone was removed en bloc.

The mean operation time (Min-Max) was 54.35 minutes (50-82) and 82.15 minutes (73-180) in PNL and LUL groups, respectively (P-value < 0.0001). Pigtail catheter remained more than 3 days in $14(35 \%)$ patients in LUL group and 12 (30\%) patients in PNL group endured ureteral catheter for more than 2 days. The average hospital stay (Min-Max) was 2.6 days (2-5) in PNL group and 3.5 days (3-8) in LUL groups ( $\mathrm{P}$ value $=0.011$ ). Stone-free status (no residual stone fragments) was complete (100\%) in both groups.

In PNL group, the average $\mathrm{Hb}$ decrease in the first postoperative day was significantly higher than in the LUL group $(0.9 \mathrm{mg} / \mathrm{dL}$ vs. $0.4 \mathrm{mg} /$
$\mathrm{dL}, \mathrm{P}$ value $=0.001)$. Regarding the need for parental pethidine to relieve the pain, there was no significant difference between the two groups ( $\mathrm{P}$ value $=0.92$ )

Except for the postoperative hemoglobin deficit, there were no statistically significant differences between the PNL and LUL patients groups in terms of other complications (Table-2). Complications included blood transfusions in 3 patients in the PNL group (one during the operation and two postoperatively), fever in 5 patients of PNL group and 4 patients of LUL group, the need for ICU in 1 patient of LUL group, and the prolonged urinary leakage in 3 patients of LUL group. The patient in LUL group who was admitted to ICU because of a respiratory insufficiency and a long operation time (180 min.) was discharged without any problem.

After calculating the hospital charges for patients in both groups according to our insurance laws, PNL procedure was found to be less expensive than LUL for the treatment of the patients with proximal ureteral stone $(\mathrm{P}-\mathrm{value}=0.0001)$. 
Table 2 - Patients' Intraoperative and postoperative data.

\begin{tabular}{lccc}
\hline Group & LUL $(\mathrm{n}=40)$ & PNL $(\mathrm{n}=40)$ & P value \\
\hline Complications & & & \\
$\quad$ Postoperative hemoglobin deficit (mg/dL) & $0.4 \mathrm{mg} / \mathrm{dL}$ & $0.9 \mathrm{mg} / \mathrm{dL}$ & 0.001 \\
$\quad$ Need for transfusion (\%) & $0(0)$ & $3(7.5)$ & 0.24 \\
$\quad$ Need for parental pethidine (mg) & $77.5(50-150)$ & $80(50-200)$ & 0.92 \\
Postoperative fever (\%) & $4(10)$ & $5(12.5)$ & 0.99 \\
$\quad$ Prolonged leakage (\%) & $3(7.5)$ & $0(0)$ & 0.24 \\
ICU admission (\%) & $1(2.5)$ & $0(0)$ & 0.99 \\
Mean operation time (minutes) & $82.15(73-180)$ & $54.35(50-82)$ & $<0.0001$ \\
Mean hospital stay (days) & $3.5(3-8)$ & $2.6(2-5)$ & 0.011 \\
Stone-free status (\%) & $40(100)$ & $40(100)$ & 1.00 \\
\hline
\end{tabular}

Performing IVU and renal ultrasound studies six months after the treatment did not revealed any residual stone, obstruction, and ureteral stenosis.

\section{DISCUSSION}

Urinary calculi are important problems in the field of urology (1). The surgical options for the treatment of proximal ureteral stones include ESWL, ureteroscopy, PNL, laparoscopic and, rarely, open surgery (2). The preferred approach for most upper-urinary stones is extracorporeal lithotripsy due to its minimal morbidity and simplicity (7). Impacted ureteral calculi larger than $1 \mathrm{~cm}$ are more resistant to ESWL due to the expansion-space theory (10). Park et al. compared the results of ESWL and ureteroscopy for ureteral stones (proximal and distal) and showed though the efficacy of ESWL decreased significantly for stones larger than $1 \mathrm{~cm}$ (83.6\% versus $42.1 \%$ ), the stone-free rate with ureteroscopic manipulation did not change by the stone size (88.9\% versus $86.6 \%$ ) (5). In the clinical guidelines of European Urological Association, it is stated that for proximal ureteral calculi $>1 \mathrm{~cm}$, ESWL and ureteroscopy have ove- rall stone free rates of 68\% (55-79) and 79\% (7187), respectively (11). Other literature shows excellent results for ureteroscopic lithotripsy using the holmium laser for proximal ureteral calculi, with a mean stone-free rate of 95\% associated with a low perforation and stricture rate of about 1\% (7). For this purpose, a better outcome could be achieved by flexible ureteroscopic lithotripsy as the primary approach (8). Although the use of holmium laser or flexible ureteroscopic lithotripsy is expensive and not available in many centers especially in developing countries, the use of pneumatic lithotripsy instead of holmium laser is not appropriate due to its high probability of stone migration (9).

Two other procedures that are suggested for proximal ureteral stone removal are laparoscopic ureterolithotomy and PNL (2). Nonetheless, the former technique requires a dissection in the retroperitoneal area to expose the ureter that is similar to open surgery, but it has a high success rate in patients with a large stone size $(2,3)$. Our previous study on 46 patients with $>1.5 \mathrm{~cm}$ upper and middle ureter stones submitted to transperitoneal laparoscopic ureterolithotomy identified $97.8 \%$ stone free rate (12). Huri et al. (13) performed transperitoneal and retroperitoneal laparos- 
copic approach in 41 patients with ureteral stones and all patients were discharged without stones. Only in one patient, the operation was converted to an open procedure. Almeida et al. (14) compared laparoscopic and open ureterolithotomy prospectively and showed lower analgesia requirements and shorter hospitalization periods in the laparoscopy group.

Furthermore, PNL does not require any special equipment and is available in most urology centers, and has the advantage of rapid stone removal. The conventional technique for PNL is in the prone position and under fluoroscopy guidance.

There are a number of options for the needle guidance during PNL, including fluoroscopy and ultrasonography (US). We used US to delineate the needle access to reduce radiation exposure. The harmful effects of x-ray are well confirmed, and for the protection against these harmful effects, highly advanced fluoroscopies and protection shields are available, although the safety is not complete. The severity of stochastic effects of x-ray is not dose dependent and may cause genetic mutation and cancer in surgeons and patients $(15,16)$. Based on the rule to use the lowest reasonably achievable radiation, the least dose has to be used.

The stone-free status of both PNL and LUL procedures were excellent (100\%) in our study. In terms of complications, there was a significant difference in the post-operative hemoglobin drop between these groups that suggests PNL may cause trauma to renal parenchyma rather than LUL. Nevertheless, some authors have shown that PNL does not result in the loss of renal function when treating renal calculi as measured by nuclear scintigraphy $(17,18)$.

In our study, some disadvantages of laparoscopic ureterolithotomy included prolonged operation time, more expensive instruments, and long hospital stay in comparison with PNL. Thus, we can suggest that ultrasonography guided PNL is a rapid and effective option for the treatment of proximal ureteral stones larger than $1 \mathrm{~cm}$. The small number of patients and the short-term follow-up periods were the limitations of this study; therefore, we recommend comparing of the two aforesaid methods with more cases under additional assessment.

\section{CONCLUSIONS}

It seems that the percutaneous nephrolithotomy under ultrasonography guidance has comparable results with laparoscopic ureterolithotomy for the treatment of the proximal ureteral stones larger than $1 \mathrm{~cm}$ with lower operation time and hospitalization period.

\section{CONFLICT OF INTEREST}

None declared.

\section{REFERENCES}

1. Lam JS, Greene TD, Gupta M: Treatment of proximal ureteral calculi: holmium:YAG laser ureterolithotripsy versus extracorporeal shock wave lithotripsy. J Urol. 2002; 167: 1972-6.

2. Hemal AK, Goel A, Goel R: Minimally invasive retroperitoneoscopic ureterolithotomy. J Urol. 2003; 169: 480-2.

3. Goel R, Aron M, Kesarwani PK, Dogra PN, Hemal AK, Gupta NP: Percutaneous antegrade removal of impacted upperureteral calculi: still the treatment of choice in developing countries. J Endourol. 2005; 19: 54-7.

4. Goel A, Hemal AK: Upper and mid-ureteric stones: a prospective unrandomized comparison of retroperitoneoscopic and open ureterolithotomy. BJU Int. 2001; 88: 679-82.

5. Park H, Park M, Park T: Two-year experience with ureteral stones: extracorporeal shockwave lithotripsy $v$ ureteroscopic manipulation. J Endourol. 1998; 12: 501-4.

6. Erhard M, Salwen J, Bagley DH: Ureteroscopic removal of mid and proximal ureteral calculi. J Urol. 1996; 155: 38-42. Erratum in: J Urol 1996; 155: 1039.

7. Mugiya S, Nagata M, Un-No T, Takayama T, Suzuki K, Fujita $\mathrm{K}$ : Endoscopic management of impacted ureteral stones using a small caliber ureteroscope and a laser lithotriptor. $J$ Urol. 2000; 164: 329-31.

8. Harmon WJ, Sershon PD, Blute ML, Patterson DE, Segura JW: Ureteroscopy: current practice and long-term complications. J Urol. 1997; 157: 28-32.

9. Karami H, Arbab AH, Hosseini SJ, Razzaghi MR, Simaei NR: Impacted upper-ureteral calculi $>1 \mathrm{~cm}$ : blind access and totally tubeless percutaneous antegrade removal or retrograde approach? J Endourol. 2006; 20: 616-9.

10. Mueller SC, Wilbert D, Thueroff JW, Alken P: Extracorporeal shock wave lithotripsy of ureteral stones: clinical experience and experimental findings. J Urol. 1986; 135: 831-4. 
11. Tiselius HG, Alken P, Buck C, Gallucci M, Knoll T, Sarica K et al.: Guidelines on urolithiasis. 2007 [cited 2007]; Available from: http://www.uroweb.org/fileadmin/user_upload/ Guidelines/Urolithiasis.pdf

12. Karami H, Javanmard B, Hasanzadeh-Hadah A, Mazloomfard MM, Lotfi B, Mohamadi R, et al.: Is It Necessary to Place a Double J Catheter After Laparoscopic Ureterolithotomy? A Four-Year Experience. J Endourol. 2012; 26: 1183-6.

13. Huri E, Basok EK, Uğurlu O, Gurbuz C, Akgül T, Ozgök Y, et al.: Experiences in laparoscopic removal of upper ureteral stones: multicenter analysis of cases, based on the TurkUroLap Group. J Endourol. 2010; 24: 1279-82.

14. Almeida GL, Heldwein FL, Graziotin TM, Schmitt CS, Telöken C: Prospective trial comparing laparoscopy and open surgery for management of impacted ureteral stones. Actas Urol Esp. 2009; 33: 1108-14.

15. Chatham JR, Dykes TE, Kennon WG, Schwartz BF: Effect of percutaneous nephrolithotomy on differential renal function as measured by mercaptoacetyl triglycine nuclear renography. Urology. 2002; 59: 522-5; discussion 525-6.
16. Moskovitz B, Halachmi S, Mullerad M, Sopov V, Burbara $\mathrm{J}$, Horev $\mathrm{N}$, et al.: Does renal function change following percutaneous nephrolithotripsy (PCNL)? Assessment with quantitative spect of Tc 99M-DSMA (QDMSA) renal scintigraphy. Eur Urol Suppl. 2005; 4: 198.

17. Wagner LK, Eifel PJ, Geise RA: Potential biological effects following high $X$-ray dose interventional procedures. J Vasc Interv Radiol. 1994; 5: 71-84.

18. Kerbl K, Clayman RV, Chandhoke PS, Urban DA, De Leo BC, Carbone JM: Percutaneous stone removal with the patient in a flank position. J Urol. 1994; 151: 686-8.

Correspondence address: Dr. Mohammad Mohsen Mazloomfard UNRC, No.103, Boostan 9th St., Pasdaran Ave. Tehran, I.R. Iran Fax: + 9821 2256-7282 Email: mazloomfard@yahoo.com 


\section{EDITORIAL COMMENT}

Ureteral lithiasis is a very frequent pathology in our country and in many occasions needs surgery. At present, endourological procedures are mostly used, with very good results and low morbidity. However, the treatment of larger stones and those located at the upper part of the ureter is challenging.

The authors present a randomized study that compared two methods of treatment for these calculi. It is a comparative, randomized study, with an adequate number of treated patients, that deserves attentions. The present guideline for the treatment of proximal ureteral calculi larger than $10 \mathrm{~mm}$ is the retrograde ureterolithotripsy. But it may have worse results if it is not used a laser lithotripsy or flexible endoscopes, and in those situations it is worthwhile the use of other surgical methods. The same gui- deline suggests the use of percutaneous ureterolithotripsy for larger calculi at the proximal ureter with 85 to $100 \%$ of success rate. The authors present the same results with the possibility of the use of ultrasonography, avoiding the use of fluoroscopy with less morbidity of the patient and medical team. Laparoscopic ureterolithotomy is becoming a more used form of treatment for these stones, with excellent results and with the same minimally invasive characteristic in relation to open surgery.

The addition of this study to literature demonstrates the possibility of use of several techniques for the treatment of larger proximal ureteral stones, according to the availability of equipment and surgeon's experience. The urologist must choose the technique that allows the complete stone removal, with less morbidity, and, if possible, with lower use of radiation.

Dr. Antonio Corrêa Lopes Neto Faculty of Medicine of $A B C$ Av. Principe de Gales, 821 Principe de Gales Santo André, SP, 09060-650, Brazil E-mail:lopes.neto@ig.com.br E-mail: lopes.neto@superig.com.br 